\title{
UNIQUENESS OF $E_{\infty}$ STRUCTURES FOR CONNECTIVE COVERS
}

\author{
ANDREW BAKER AND BIRGIT RICHTER
}

(Communicated by Paul Goerss)

\begin{abstract}
We refine our earlier work on the existence and uniqueness of $E_{\infty}$ structures on $K$-theoretic spectra to show that the connective versions of real and complex $K$-theory as well as the connective Adams summand $\ell$ at each prime $p$ have unique structures as commutative $\mathbb{S}$-algebras. For the $p$ completion $\ell_{p}$ we show that the McClure-Staffeldt model for $\ell_{p}$ is equivalent as an $E_{\infty}$ ring spectrum to the connective cover of the periodic Adams summand $L_{p}$. We establish a Bousfield equivalence between the connective cover of the Lubin-Tate spectrum $E_{n}$ and $B P\langle n\rangle$.
\end{abstract}

\section{INTRODUCTION}

The aim of this short note is to establish the uniqueness of $E_{\infty}$ structures on connective covers of certain periodic commutative $\mathbb{S}$-algebras $E$, most prominently for the connective $p$-complete Adams summand. It is clear that the connective cover of an $E_{\infty}$ ring spectrum inherits an $E_{\infty}$ structure; there is even a functorial way of assigning a connective cover within the category of $E_{\infty}$ ring spectra [10, VII.4.3]. But it is not obvious in general that this $E_{\infty}$ multiplication is unique.

Our main concern is with examples in the vicinity of $K$-theory; we apply our uniqueness theorem to real and complex $K$-theory and their localizations and completions and to the Adams summand and its completions.

The existence and uniqueness of $E_{\infty}$ structures on the periodic spectra $K U$, $K O$ and $L$ was established in [5] by means of the obstruction theory for $E_{\infty}$ structures developed by Goerss and Hopkins [9] and Robinson [13]. Note however, that obstruction-theoretic methods would fail in the connective cases. Let $e$ be a commutative ring spectrum. If $e$ satisfies some Künneth and universal coefficient properties [13, proposition 5.4], then the obstruction groups for $E_{\infty}$ multiplications consist of André-Quillen cohomology groups in the context of differential graded $E_{\infty}$ algebras applied to the graded commutative $e_{*}$-algebra $e_{*} e$. Besides problems with non-projectivity of $e_{*} e$ over $e_{*}$, the algebra structures of $k u_{*} k u, k o_{*} k o$ and $\ell_{*} \ell$ are far from being étale, and therefore one would obtain nontrivial obstruction groups. One would then have to identify actual obstruction classes in these obstruction groups in order to establish the uniqueness of the given $E_{\infty}$ structure,

Received by the editors October 10, 2006 and, in revised form, October 25, 2006.

2000 Mathematics Subject Classification. Primary 55P43, 55N15; Secondary 19L41.

The first author thanks the Max-Planck Institute and the mathematics department in Bonn.

The second author was partially supported by the Strategisk Universitetsprogram i Ren Matematikk (SUPREMA) of the Norwegian Research Council. 
but at the moment, this seems to be an intractable problem. Thus an alternative approach is called for.

In Theorem 1.3 we prove that a unique $E_{\infty}$ structure on $E$ gives rise to a unique structure on the connective cover for spectra $E$ which are obtained from some connective spectrum via a process of Bousfield localization and which satisfy some mild homotopical conditions (see Assumption 1.2). In particular, we identify the $E_{\infty}$ structure on the $p$-completed connective Adams summand $\ell_{p}$ provided by McClure and Staffeldt in [11] with the one that arises by taking the unique $E_{\infty}$ structure on the periodic Adams summand $L=E(1)$ developed in [5] and taking its connective cover.

Our theorem applies as well to the connective covers of the Lubin-Tate spectra $E_{n}$, and we prove in section 3 that these spectra are Bousfield equivalent to the truncated Brown-Peterson spectra $B P\langle n\rangle$. Unlike other spectra that are Bousfield equivalent to $B P\langle n\rangle$, such as the connective cover of the completed Johnson-Wilson

spectrum, $\widehat{E(n)}$, the connective cover of $E_{n}$ is calculationally convenient in the sense that its coefficients (see (3.1)) are rather small. So far, only $B P\langle 1\rangle=\ell$ is known to have an $E_{\infty}$ structure, and we propose the connective cover of $E_{n}$ as an $E_{\infty}$ approximation of $B P\langle n\rangle$.

\section{1. $E_{\infty}$ STRUCTURES ON CONNECTIVE COVERS}

We will give some background on three standard constructions, which we will need later: namely, the connective covers functor, the bar construction that turns an $E_{\infty}$-ring spectrum into a commutative $\mathbb{S}$-algebra and Bousfield localization.

- May et al. constructed a connective cover functor in [10, VII.3.2], which we denote by $c(-)$. For every $E_{\infty}$ ring spectrum $E, c(E)$ is a connective $E_{\infty}$ ring spectrum $E$ which depends functorially on $E$ and which comes with a morphism of $E_{\infty}$ ring spectra $c(E) \stackrel{\varepsilon}{\rightarrow} E$ [10, VII.4.3]. This map induces an isomorphism on homotopy groups in nonnegative degrees.

- For any $E_{\infty}$ ring spectrum $E$, there is a weakly equivalent commutative S-algebra $B(E)$, with an equivalence

$$
\lambda: B(E) \stackrel{\rho}{\rightarrow} E,
$$

in the $E_{\infty}$ category [8, II.3.6].

- For a commutative $\mathbb{S}$-algebra $R$ and an $R$-module $M$, we let $L_{M}^{R}(-)$ denote Bousfield localization at $M$ in the category of $R$-modules and we denote the localization map by $\sigma: E \longrightarrow L_{M}^{R}(E)$ for any $R$-module $E$ [8, chapter VIII]. The case that will be most relevant to us is $M=R\left[X^{-1}\right]$, where an element $X \in \pi_{*} R$ is inverted. The case $R=\mathbb{S}$ corresponds to ordinary Bousfield localization.

A ring spectrum for us is a homotopy notion, i.e., it is an object in the homotopy category of spectra with a monoid structure in the homotopy category. We will use the following notion of uniqueness for $E_{\infty}$ structures.

Definition 1.1. An $E_{\infty}$ structure on some homotopy commutative and associative ring spectrum $E$ is unique if the following holds. For every $E_{\infty}$ ring spectrum $E^{\prime}$ and every map of ring spectra $\varphi: E^{\prime} \longrightarrow E$ that induces an isomorphism on homotopy groups, there is a morphism in the homotopy category of $E_{\infty}$ ring spectra $\varphi^{\prime}: E^{\prime} \longrightarrow E$ such that $\varphi^{\prime}$ is homotopic to $\varphi$ as maps of ring spectra. 
In the appendix to this paper, we relate this notion to the one used in obstruction theory.

For the rest of the paper we assume the following.

Assumption 1.2. Let $E$ be a periodic commutative $\mathbb{S}$-algebra with periodicity element $v \in E_{*}$ of positive degree. We assume that $E$ is obtained from a connective commutative $\mathbb{S}$-algebra $e$ by Bousfield localization at $e\left[v^{-1}\right]$ in the category of $e$ modules, i.e., $E=L_{e\left[v^{-1}\right]}^{e}(e)=e\left[v^{-1}\right]$. Furthermore we assume that the localization map induces an isomorphism between the homotopy groups of $e$ and the homotopy groups of the connective cover $c(E)$ of $E$ and that $E$ satisfies a universal coefficient theorem.

Theorem 1.3. Assume that we know that the $E_{\infty}$ structure on $E$ is unique. Then the $E_{\infty}$ structure on $c(E)$ is unique.

Proof. Each commutative $\mathbb{S}$-algebra can be viewed as an $E_{\infty}$ ring spectrum. Let $e^{\prime}$ be a model for the connective cover $c(E)$, i.e., $e^{\prime}$ is an $E_{\infty}$ ring spectrum with a map of ring spectra $\varphi$ to $c(E)$, such that $\pi_{*}(\varphi)$ is an isomorphism. Write $v \in e_{*}^{\prime}$ for the isomorphic image of $v$ under the inverse of $\pi_{*}(\varphi)$. The universal property of $e\left[v^{-1}\right][8, \mathrm{~V} .1 .13]$ asserts that the ring map $\varepsilon \circ \varphi \circ \rho: B\left(e^{\prime}\right) \longrightarrow E=e\left[v^{-1}\right]$ gives rise to a map from $B\left(e^{\prime}\right)\left[v^{-1}\right]$ to $E$. Due to the presence of a universal coefficient theorem for $E$, the homotopy classes of maps from $B\left(e^{\prime}\right)\left[v^{-1}\right]$ to $E$ are in bijective correspondence to the $E_{*}$-module maps from $E_{*}\left(B\left(e^{\prime}\right)\left[v^{-1}\right]\right)$ to $E_{*}$. As we started with a ring map $\varphi$, we obtain a map of $E_{*}$-algebras from $E_{*}\left(B\left(e^{\prime}\right)\left[v^{-1}\right]\right)$ to $E_{*}$, and this corresponds to a map of ring spectra from $B\left(e^{\prime}\right)\left[v^{-1}\right]$ to $E$.

As the $E_{\infty}$ structure on $E$ is unique by assumption, this ring map can be replaced by an equivalent equivalence, $\xi$, of $E_{\infty}$ ring spectra. We consider the following diagram whose dotted lines provide a zigzag of $E_{\infty}$ equivalences and hence a map in the homotopy category of $E_{\infty}$ ring spectra.

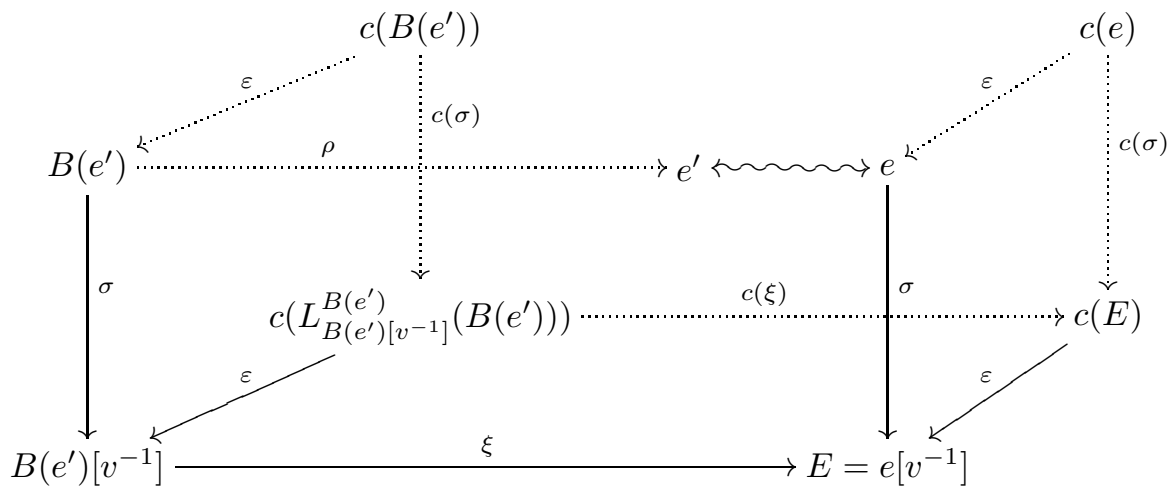

Real and complex $K$-theory, $k o$ and $k u$, have $E_{\infty}$ structures obtained using algebraic $K$-theory models [10, VIII, §2]. The connective Adams summand $\ell$ has an $E_{\infty}$ structure because it is the connective cover of the Johnson-Wilson spectrum $E(1)$ with $E(1)_{*}=\mathbb{Z}_{(p)}\left[v_{1}^{ \pm}\right],\left|v_{1}\right|=2 p-2$. In the following we will refer to these models as the standard ones. The $E_{\infty}$ structures on $K O, K U$ and $E(1)$ are unique by $[5$, theorems $7.2,6.2]$. In all of these cases, the periodic versions are obtained by Bousfield localization [8, VIII.4.3]. 
Corollary 1.4. The $E_{\infty}$ structures on $k o, k u$ and $\ell$ are unique.

\section{The $p$-Complete connective Adams summand}

In [11], McClure and Staffeldt construct a model for the $p$-completed connective Adams summand using the algebraic $K$-theory of fields. Let $\widetilde{\ell}=K\left(\mathbf{k}^{\prime}\right)$, the algebraic $K$-theory spectrum of $\mathbf{k}^{\prime}=\bigcup_{i} \mathbb{F}_{q^{p^{i}}}$, where $q$ is a prime which generates the $p$-adic units $\mathbb{Z}_{p}^{\times}$. Then the $p$-completion of $\tilde{\ell}$ is additively equivalent to the $p$-completed connective Adams summand $\ell_{p}$ [11, proposition 9.2]. For further details, see also $[2, \S 2]$. Note that the $p$-completion $\ell_{p}$ inherits an $E_{\infty}$ structure from $\ell$ because $p$-completion is Bousfield localization with respect to the $\bmod p$ Moore spectrum [7, proposition 2.5] and therefore preserves commutative $\mathbb{S}$-algebra structures [8, VIII.2.2].

An a priori different model for the $p$-completion of the connective Adams summand can be obtained by taking the connective cover of the $p$-complete periodic version $L=E(1)$. For the following we denote the composition $B \circ c$ by $\bar{c}$.

Note that $p$-completion and Bousfield localization of $\ell$ in the category of $\ell$ modules with respect to $L$ are compatible in the following sense. Consider $\ell=\bar{c}(L)$ and its $p$-completion

$$
\lambda_{\ell}: \ell \longrightarrow \ell_{p}=(\bar{c}(L))_{p} .
$$

The $p$-completion map $\lambda$ is functorial in the spectrum; therefore the following diagram of solid arrows commutes.

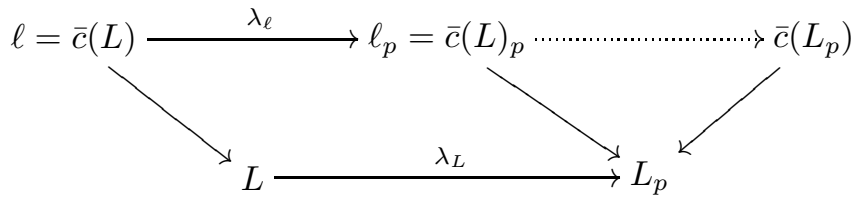

The universal property of the connective cover functor ensures that there is a map in the homotopy category of commutative $\mathbb{S}$-algebras from $\ell_{p}$ to $\bar{c}\left(L_{p}\right)$ which is a weak equivalence. In the following we will no longer distinguish $\ell_{p}$ from $\bar{c}\left(L_{p}\right)$ and will denote this model simply by $\ell_{p}$.

Proposition 2.1. The McClure-Staffeldt model $\tilde{\ell}_{p}$ of the p-complete connective Adams summand is equivalent as an $E_{\infty}$ ring spectrum to $\ell_{p}$.

Remark 2.2. If $E$ is a commutative $\mathbb{S}$-algebra with naive $G$-action for some group $G$, then neither the connective cover functor $\bar{c}(-)$ nor Bousfield localization of $E$ has to commute with taking homotopy fixed points. As an example, consider connective complex $K$-theory $k u$ with the conjugation action by $C_{2}$. The homotopy fixed points $k u^{h C_{2}}$ are not equivalent to $k o$, but on the periodic versions we obtain $K U^{h C_{2}} \simeq K O$.

Proof of Proposition 2.1. Consider the algebraic $K$-theory model for connective complex $K$-theory, $k u=K(\mathbf{k})$, with $\mathbf{k}=\bigcup_{i} \mathbb{F}_{q^{p^{i}(p-1)}}$. The canonical inclusions $\mathbb{F}_{q^{p^{i}}} \hookrightarrow \mathbb{F}_{q^{p^{i}(p-1)}}$ assemble into a map $j: \mathbf{k}^{\prime} \longrightarrow \mathbf{k}$. The Galois group $C_{p-1}$ of $\mathbf{k}$ over $\mathbf{k}^{\prime}$ acts on $\mathbf{k}$ and induces an action on algebraic $K$-theory. As $\mathbf{k}^{\prime}$ is fixed under the 
action of $C_{p-1}$ there is a factorization of $K(j)_{p}$ as

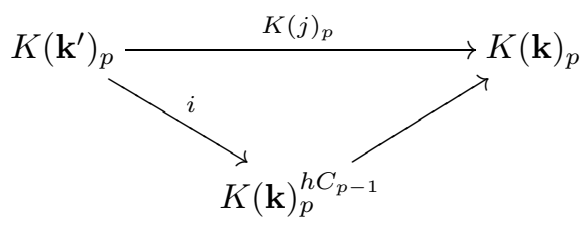

and $i$ yields a weak equivalence of commutative $\mathbb{S}$-algebras, where $K(\mathbf{k})_{p}^{h C_{p-1}}$ is a model for the connective $p$-complete Adams summand which is weakly equivalent to $\widetilde{\ell}_{p}$ (see $\left.[2, \S 2]\right)$.

Consider the composition of the following chain of maps between commutative S-algebras:

$$
K\left(\mathbf{k}^{\prime}\right)_{p} \stackrel{i}{\longrightarrow}\left(K(\mathbf{k})_{p}\right)^{h C_{p-1}} \longrightarrow K(\mathbf{k})_{p} \longrightarrow K U_{p} .
$$

The target $K U_{p}$ is as well the target of the map $\bar{c}\left(K U_{p}\right) \longrightarrow K U_{p}$. Note that the universal property of $\bar{c}(-)$ yields a zigzag $\varsigma: K(\mathbf{k})_{p} \leadsto \bar{c}\left(K U_{p}\right)$ of equivalences between $K(\mathbf{k})_{p}$ and $\bar{c}\left(K U_{p}\right)$ in the category of commutative $\mathbb{S}$-algebras.

As $K U_{p}$ is the Bousfield localization of $K(\mathbf{k})_{p}$ in the category of $K(\mathbf{k})_{p}$-modules with respect to the Bott element,

$$
K U_{p}=L_{K(\mathbf{k})_{p}\left[\beta^{-1}\right]}^{K(\mathbf{k})_{p}} K(\mathbf{k})_{p},
$$

it inherits the $C_{p-1}$-action on $K(\mathbf{k})_{p}$. The functoriality of the connective cover lifts this action to an action on $\bar{c}\left(K U_{p}\right)$.

The connective cover functor is in fact a functor in the category of commutative $\mathbb{S}$-algebras with multiplicative naive $G$-action for any group $G$. To see this we have to show that the map $\bar{c}(A) \longrightarrow A$ is $G$-equivariant if $A$ is a commutative $\mathbb{S}$-algebra with an underlying naive $G$-spectrum. The functor $B(-)$ does not cause any problems. Proving the claim for the functor $c$ involves chasing the definition given in [10, VII, §3].

The prespectrum underlying $c(A)$ applied to an inner product space $V$ is defined as $T\left(A_{0}\right)(V)$, where $A_{0}$ is the zeroth space of the spectrum $A$ and $T$ is a certain bar construction involving suspensions and a monad consisting of the product of a fixed $E_{\infty}$ operad with the partial operad of little convex bodies $\mathcal{K}$. For a fixed $V$ the suspension $\Sigma^{V}$ and the operadic term $\mathcal{K}_{V}$ are used. As the $G$-action is compatible with the $E_{\infty}$ and the additive structure of $A$, the evaluation map $T\left(A_{0}\right)(V) \longrightarrow A(V)$ is $G$-equivariant. For varying $V$, these maps constitute a map of prespectra and its adjoint on the level of spectra is $c(A) \longrightarrow A$. As the spectrification functor preserves $G$-equivariance, the claim follows. Therefore the resulting zigzag $\varsigma: K(\mathbf{k})_{p} \leadsto \bar{c}\left(K U_{p}\right)$ is $C_{p-1}$-equivariant, and we obtain an induced zigzag on homotopy fixed points,

$$
\varsigma^{h C_{p-1}}:\left(K(\mathbf{k})_{p}\right)^{h C_{p-1}} \leftrightarrow\left(\bar{c}\left(K U_{p}\right)\right)^{h C_{p-1}} .
$$

As $\varsigma$ is an isomorphism in the homotopy category and is $C_{p-1}$-equivariant, $\varsigma^{h C_{p-1}}$ yields an isomorphism as well.

\section{Connective Lubin-Tate spectra}

Goerss and Hopkins proved in [9] that the Lubin-Tate spectra $E_{n}$ with

$$
\left(E_{n}\right)_{*}=W\left(\mathbb{F}_{p^{n}}\right)\left[\left[u_{1}, \ldots, u_{n-1}\right]\right]\left[u^{ \pm 1}\right] \quad \text { with }\left|u_{i}\right|=0 \text { and }|u|=-2
$$


possess unique $E_{\infty}$ structures for all primes $p$ and all $n \geqslant 1$. The connective cover $c\left(E_{n}\right)$ has coefficients

$$
\left(c\left(E_{n}\right)\right)_{*}=W\left(\mathbb{F}_{p^{n}}\right)\left[\left[u_{1}, \ldots, u_{n-1}\right]\right]\left[u^{-1}\right] \quad \text { with }\left|u_{i}\right|=0 \text { and }|u|=-2 .
$$

Of course $\bar{c}\left(E_{n}\right)\left[\left(u^{-1}\right)^{-1}\right] \sim E_{n}$.

The spectra $B P\langle n\rangle$ can be built from the Brown-Peterson spectrum $B P$ by killing all generators of the form $v_{m}$ with $m>n$ in $B P_{*}=\mathbb{Z}_{(p)}\left[v_{1}, v_{2}, \ldots\right]$. Using for instance Angeltveit's result [1, theorem 4.2], one can prove that the $B P\langle n\rangle$ are $A_{\infty}$ spectra and from [4] it is known that this $\mathbb{S}$-algebra structure can be improved to an $M U$-algebra structure. On the other hand, Strickland showed in [14] that $B P\langle n\rangle$ with $n \geqslant 2$ is not a homotopy commutative $M U$-ring spectrum for $p=2$. We offer $c\left(E_{n}\right)$ as a replacement for the $p$-completion $B P\langle n\rangle_{p}$ of $B P\langle n\rangle$.

We also need to recall that in the category of $M U$-modules, $E(n)$ is the Bousfield localization of $B P\langle n\rangle$ with respect to $B P\langle n\rangle\left[v_{n}^{-1}\right]$; hence by [8] it inherits the structure of an $M U$-algebra and the natural map $B P\langle n\rangle \longrightarrow E(n)$ is a morphism of $M U$-algebras. Furthermore, the Bousfield localization of $E(n)$ with respect to the $M U$-algebra $K(n)$ is the $I_{n}$-adic completion $\widehat{E(n)}$, which was shown to be a commutative $\mathbb{S}$-algebra in [5], and the natural map $\widehat{E(n)} \longrightarrow E_{n}$ is a morphism of commutative $\mathbb{S}$-algebras; see for example [6, example 2.2.9]. Thus there is a morphism of ring spectra $B P\langle n\rangle \longrightarrow E_{n}$ that lifts to a map $B P\langle n\rangle \longrightarrow c\left(E_{n}\right)$ in the homotopy category.

Proposition 3.1. The spectra $B P\langle n\rangle$ and $B P\langle n\rangle_{p}$ are Bousfield equivalent to $c\left(E_{n}\right)$.

Proof. On coefficients, we obtain a ring homomorphism $\left(B P\langle n\rangle_{p}\right)_{*} \longrightarrow\left(c\left(E_{n}\right)\right)_{*}$, which is given by

$$
v_{k} \longmapsto \begin{cases}u^{1-p^{k}} u_{k} & \text { for } 1 \leqslant k \leqslant n-1, \\ u^{1-p^{n}} & \text { for } k=n,\end{cases}
$$

extending the natural inclusion of the $p$-adic integers $\mathbb{Z}_{p}=W\left(\mathbb{F}_{p}\right)$ into $W\left(\mathbb{F}_{p^{n}}\right)$. This homomorphism is induced by a map of ring spectra.

Recall from [3] that $E(n)$ and $\widehat{E(n)}$ are Bousfield equivalent as $\mathbb{S}$-modules, and it follows that $E_{n}$ is Bousfield equivalent to these since it is a finite wedge of suspensions of $\widehat{E(n)}$.

If $X$ is a $p$-local spectrum with torsion free homotopy groups, then its $p$-completion $X_{p}$ is Bousfield equivalent to $X$, i.e., $\left\langle X_{p}\right\rangle=\langle X\rangle$. This follows using the cofibre triangles (in which $M(p)$ is the $\bmod p$ Moore spectrum and the circled arrow indicates a map of degree one)
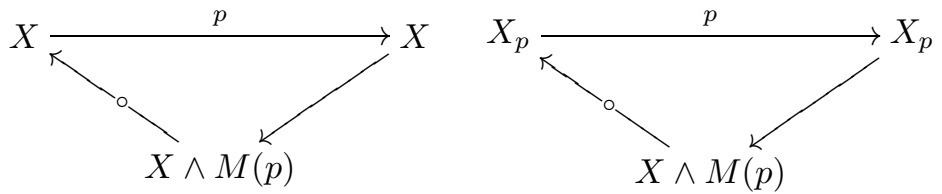

together with the fact that the rationalization $p^{-1} X$ is a retract of $p^{-1}\left(X_{p}\right)$. In particular, we have $\left\langle B P\langle n\rangle_{p}\right\rangle=\langle B P\langle n\rangle\rangle$ and $\left\langle E(n)_{p}\right\rangle=\langle E(n)\rangle$.

From [12, theorem 2.1], the Bousfield class of $B P\langle n\rangle$ is

$$
\langle B P\langle n\rangle\rangle=\langle E(n)\rangle \vee\left\langle H \mathbb{F}_{p}\right\rangle .
$$


There is a cofibre triangle

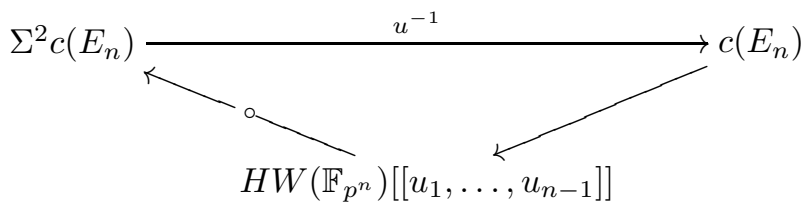

in which $H W\left(\mathbb{F}_{p^{n}}\right)\left[\left[u_{1}, \ldots, u_{n-1}\right]\right]$ is the Eilenberg-Mac Lane spectrum. More generally we can construct a family of Eilenberg-Mac Lane spectra with $W\left(\mathbb{F}_{p^{n}}\right)\left[\left[u_{1}, \ldots, u_{k}\right]\right]$ as coefficients for $k=0, \ldots, n-1$ which are related by cofibre triangles

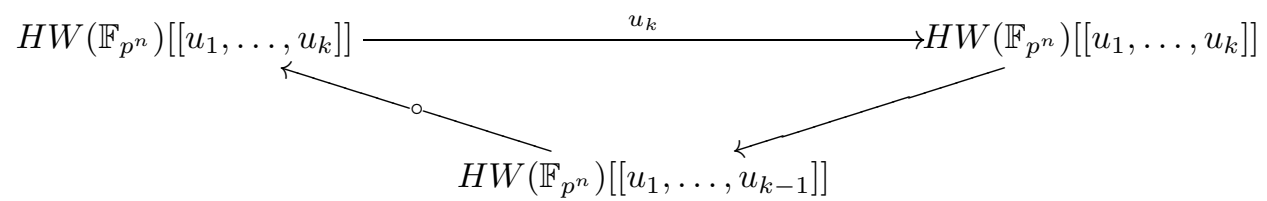

such that for $k=0$ we obtain $H W\left(\mathbb{F}_{p^{n}}\right)$. With the help of these cofibre sequences we can identify $\left\langle H W\left(\mathbb{F}_{p^{n}}\right)\left[\left[u_{1}, \ldots, u_{k}\right]\right]\right\rangle$ with $\left\langle H W\left(\mathbb{F}_{p^{n}}\right)\left[\left[u_{1}, \ldots, u_{k-1}\right]\right]\right\rangle \vee$ $\left\langle H W\left(\mathbb{F}_{p^{n}}\right)\left[\left[u_{1}, \ldots, u_{k}\right]\right]\left[u_{k}^{-1}\right]\right\rangle$.

In general, if $R$ is a commutative ring, then the ring of finite tailed Laurent series $R((x))$ is faithfully flat over $R$, and therefore we have

$$
\langle H R((x))\rangle=\langle H R\rangle .
$$

Using this auxiliary fact we inductively get that

$$
\left\langle H W\left(\mathbb{F}_{p^{n}}\right)\left[\left[u_{1}, \ldots, u_{k}\right]\right]\right\rangle=\left\langle H W\left(\mathbb{F}_{p^{n}}\right)\left[\left[u_{1}, \ldots, u_{k-1}\right]\right]\right\rangle .
$$

This reduces the Bousfield class of $c\left(E_{n}\right)$ to $\left\langle E_{n}\right\rangle \vee\left\langle H W\left(\mathbb{F}_{p^{n}}\right)\right\rangle$. As $W\left(\mathbb{F}_{p^{n}}\right)$ is a finitely generated free $\mathbb{Z}_{p}$-module and as $\left\langle H \mathbb{Z}_{p}\right\rangle=\langle H \mathbb{Q}\rangle \vee\left\langle H \mathbb{F}_{p}\right\rangle$, this leads to

$$
\begin{aligned}
\left\langle c\left(E_{n}\right)\right\rangle & =\left\langle E(n) \vee H \mathbb{Q} \vee H \mathbb{F}_{p}\right\rangle \\
& =\left\langle E(n) \vee H \mathbb{F}_{p}\right\rangle=\langle B P\langle n\rangle\rangle .
\end{aligned}
$$

\section{Appendix: Uniqueness and $E_{\infty}$-Mapping SPACES}

If $E$ and $F$ are spectra whose $E_{\infty}$ structure was provided by the obstruction theory of Goerss and Hopkins [9], then we can compare our uniqueness notion with theirs. Note that examples of such $E_{\infty}$ ring spectra include ring spectra such as $E_{n}[9,7.6], K O, K U, L$ and $\widehat{E(n)}[5]$. In such cases the Hurewicz map

$$
\pi_{0} \operatorname{Hom}_{E_{\infty}}(E, F) \stackrel{h}{\longrightarrow} \operatorname{Hom}_{F_{*}-\operatorname{alg}}\left(F_{*} E, F_{*}\right)
$$

from the connected components of the derived space of $E_{\infty}$-maps between $E$ and $F$ and the $F_{*}$-algebra homomorphisms from $F_{*} E$ to $F_{*}$ is a bijection [9, corollary 4.4, theorem 4.5]. Assume that we have a mere ring map $\varphi$ as above between $E$ and $F$. This gives rise to a map of $F_{*}$-algebras from $F_{*} E$ to $F_{*}$ by composing $F_{*}(\varphi)$ with the multiplication $\mu$ in $F_{*} F$. In the presence of a universal coefficient theorem we have $\operatorname{Hom}_{F_{*}-\operatorname{hom}}\left(F_{*} E, F_{*}\right)=[E, F]$; therefore the element $\mu \circ F_{*}(\varphi)$ gives rise to a homotopy class of maps of ring spectra $\widetilde{\varphi}$ from $E$ to $F$. We can assume that we have a functorial cofibrant replacement $Q(-)$; hence we obtain a ring map $Q(\widetilde{\varphi})$ from 
$Q(E)$ to $Q(F)$. Via the isomorphism of (4.1) this gives a map $\Phi$, of $E_{\infty}$ spectra from $Q(E)$ to $Q(F)$; therefore we obtain a zigzag

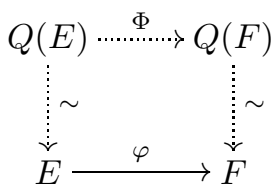

of weak equivalences of $E_{\infty}$ spectra from $E$ to $F$. Thus in such cases our definition agrees with the uniqueness notion that is natural in the Goerss-Hopkins setting.

\section{ACKNOWLEDGMENT}

We are grateful to John Rognes who suggested exploiting the functoriality of the connective cover functor to obtain uniqueness of $E_{\infty}$ structures.

\section{REFERENCES}

1. V. Angeltveit, $A_{\infty}$-obstruction theory and the strict associativity of $E / I$, preprint (2004); http://hopf .math.purdue.edu/cgi-bin/generate?/Angeltveit/Ainfinity

2. C. Ausoni, Topological Hochschild homology of connective complex K-theory, Amer. J. Math. 127 (2005) 1261-1313. MR2183525 (2006k:55016)

3. A. Baker, $I_{n}$-local Johnson-Wilson spectra and their Hopf algebroids, Documenta Math. 5 (2000) 351-364. MR1767568 (2001k:55012)

4. A. Baker \& A. Jeanneret, Brave new Hopf algebroids and extensions of MU-algebras, Homology, Homotopy and Applications 4 (2002) 163-173. MR1937961 (2003g:55012)

5. A. Baker \& B. Richter, $\Gamma$-cohomology of rings of numerical polynomials and $E_{\infty}$ structures on K-theory, Comment. Math. Helv. 80 (4) (2005) 691-723. MR2182697 (2006i:55012)

6. A. Baker \& B. Richter, Realizability of algebraic Galois extensions by strictly commutative ring spectra, Trans. Amer. Math. Soc. 359 (2007), 827-857. MR2255198

7. A. K. Bousfield, The localization of spectra with respect to homology, Topology 18 (1979) 257-281. MR551009 (80m:55006)

8. A. Elmendorf, I. Kriz, M. Mandell \& J. P. May, Rings, modules, and algebras in stable homotopy theory, Mathematical Surveys and Monographs 47 (1997). MR1417719 (97h:55006)

9. P. G. Goerss \& M. J. Hopkins, Moduli spaces of commutative ring spectra, in 'Structured Ring Spectra', eds. A. Baker \& B. Richter, London Math. Soc. Lecture Notes, 315, Cambridge University Press (2004) 151-200. MR2125040 (2006b:55010)

10. J. P. May, $E_{\infty}$ ring spaces and $E_{\infty}$ ring spectra, With contributions by F. Quinn, N. Ray \& J. Tornehave, Lecture Notes in Mathematics 577 (1977). MR0494077 (58:13008)

11. J. E. McClure \& R. E. Staffeldt, On the topological Hochschild homology of bu, I, Amer. J. Math. 115 (1993) 1-45. MR1209233 (94d:55020)

12. D. C. Ravenel, Localization with respect to certain periodic homology theories, Amer. J. Math. 106 (1984) 351-414. MR737778 (85k:55009)

13. A. Robinson, Gamma homology, Lie representations and $E_{\infty}$ multiplications, Invent. Math. 152 (2003) 331-348. MR1974890 (2004c:55020)

14. N. Strickland, Products on MU-modules, Trans. Amer. Math. Soc. 351 (1999) 2569-2606. MR1641115 (2000b:55003)

Department of Mathematics, University of Glasgow, Glasgow G12 8QW, Scotland E-mail address: a.baker@maths.gla.ac.uk $U R L:$ http://www.maths.gla.ac.uk/ ajb

Department Mathematik der Universität Hamburg, 20146 Hamburg, Germany

E-mail address: richter@math.uni-hamburg.de

$U R L:$ http://www.math.uni-hamburg.de/home/richter/ 